\title{
Population based study of late onset cerebellar ataxia in south east Wales
}

\author{
M B Muzaimi, J Thomas, S Palmer-Smith, L Rosser, P S Harper, C M Wiles, D Ravine, N P Robertson
}

J Neurol Neurosurg Psychiatry 2004;75:1129-1134. doi: 10.1136/jnnp.2003.014662

See end of article for authors' affiliations

\section{Correspondence to:}

Dr N P Robertson, Section of Neurology, Department of Medicine, University Hospital of Wales, Heath Park, Cardiff CF14 4XN, UK; robertsonnp@cardiff. ac.uk

Received 13 March 2003 In revised form

16 October 2003

Accepted

1 November 2003

\begin{abstract}
Objective: To determine the prevalence and causation of late onset cerebellar ataxia (LOCA) in south east Wales, United Kingdom.

Methods: A population based study of LOCA was conducted in a defined geographical region with a total population of 742 400. Multiple sources of ascertainment were used to identify all cases prevalent on 1 January 2001. The inclusion criteria were: a predominantly progressive cerebellar ataxia with onset of symptoms at age $\geqslant 18$ years; and disease duration of $\geqslant 1$ year. Cases with known acquired ataxias, ataxic syndromes with associated prominent autonomic dysfunction and/or atypical parkinsonism suggestive of multiple system atrophy and disorders with ataxia as a minor feature were excluded.

Results: We identified 76 index cases of LOCA, of whom 63 were sporadic, idiopathic LOCA (ILOCA) and 13 were familial LOCA, of whom six had either spinocerebellar ataxia type 6, Friedreich's ataxia or dominant episodic ataxia. The mean annual incidence rate for the period 1999-2001 was 0.3/100 000 population/year. The crude prevalence rates were 8.4 per 100000 (95\% Cl 7.2 to 11.6 ) for ILOCA and 1.8 per 100000 (95\% Cl 0.8 to 2.7) for inherited LOCA. Of the 54/63 (85.7\%) patients with ILOCA who were assessed, mean (SD) age at onset of symptoms was 53.8 (14.1) years (range 19 to 78) with a male:female ratio of 2.1:1. The mean disease duration was 8.7 (6.3) years (range 1 to 31). The most frequent presenting complaint was disturbance in gait (90.7\%). One-third had a relatively pure cerebellar syndrome (33.3\%) and two-thirds (66.7\%) had additional extracerebellar neurological features. The majority (92\%) were ambulant but only $9.3 \%$ were independently self-caring.

Conclusion: This population based study provides insight into LOCA within a defined region and will inform decisions about the rational use of healthcare resources for patients with LOCA.
\end{abstract}

$\mathrm{T}^{\mathrm{t}}$ he ataxias are clinically, pathologically, and aetiologically a heterogeneous group of disorders that result in considerable health burden. ${ }^{12}$ The widely variable and overlapping symptoms and signs, often with additional extracerebellar neurological involvement, complicate the clinical assessment. ${ }^{1}$ Consequently, adult patients presenting predominantly with symptoms and signs of late onset cerebellar ataxia (LOCA) may impose diagnostic difficulties.

LOCA can be categorised into acquired, hereditary, and non-hereditary degenerative ataxias. ${ }^{2}$ Recent advances in molecular genetics have indicated that up to $60 \%$ of familial cases and $19 \%$ of sporadic cases may have a genetic basis. ${ }^{3-7}$ As a result, increasing batteries of tests are being used in cases of LOCA to screen for rare causes, albeit at a considerable cost. However, there is a further group of idiopathic cases (ILOCA). ${ }^{5-9}$ A recent study has suggested that a proportion of these patients may have multiple system atrophy (MSA), ${ }^{10}$ a disorder characterised clinically by progressive ataxia with an almost exclusive association with autonomic dysfunction and at autopsy by presence of oligodendroglial intracytoplasmic inclusions. ${ }^{11}$ The increasing availability of molecular genetic diagnostic tests has heightened interest in the hereditary contribution to LOCA in epidemiological investigations worldwide, especially for the autosomal dominant spinocerebellar ataxia (SCA) subtypes. ${ }^{42-21}$ These have reported wide variations in prevalence of SCA genotypes both geographically and between ethnic groups. It is likely that these variations reflect founder effects, although selective laboratory based sampling may contribute. There are few data concerning the overall prevalence and aetiology of LOCA based on population surveys and relevant prognostic information is even more scarce. Previously reported estimates from isolated and selected populations have yielded prevalence for hereditary ataxias of between 0.31 and 41 per $100000 . .^{22-27}$ No recent comparable population based information for LOCA is available for the United Kingdom (UK). To overcome this deficiency, we conducted a population based study into causation and prevalence of LOCA in south east Wales, a region in which a well-established neuroepidemiological framework already exists. ${ }^{28-31}$

\section{METHODS}

\section{Study population}

The residents of Bro Taf Health Authority (BTHA) in south east Wales, UK constituted the population base for the study (fig 1). This is considered to be a relatively stable population, estimated at 742400 of whom $570000(76 \%)$ are aged $\geqslant 18$ years with net civilian migration and other changes of $0.4 \%$ per annum. ${ }^{32}$ There are 134 general practices within the health authority boundaries but a number of these also serve neighbouring regions. Individuals who were alive and normally resident within BTHA areas on l January 2001 were considered prevalent cases.

\section{Case definition}

The clinical classification and inclusion criteria were (i) age at onset of symptoms $\geqslant 18$ years and (ii) a predominantly progressive cerebellar ataxia with disease duration of $\geqslant 1$ year (defined from age at onset to the prevalent date). Cases were

Abbreviations: $A D L$, activities of daily living; BTHA, Bro Taf Health Authority; FRDA, Friedreich's ataxia; GP, general practitioner; LOCA, late onset cerebellar ataxia; MSA, multiple system atrophy; SCA, spinocerebellar ataxia 


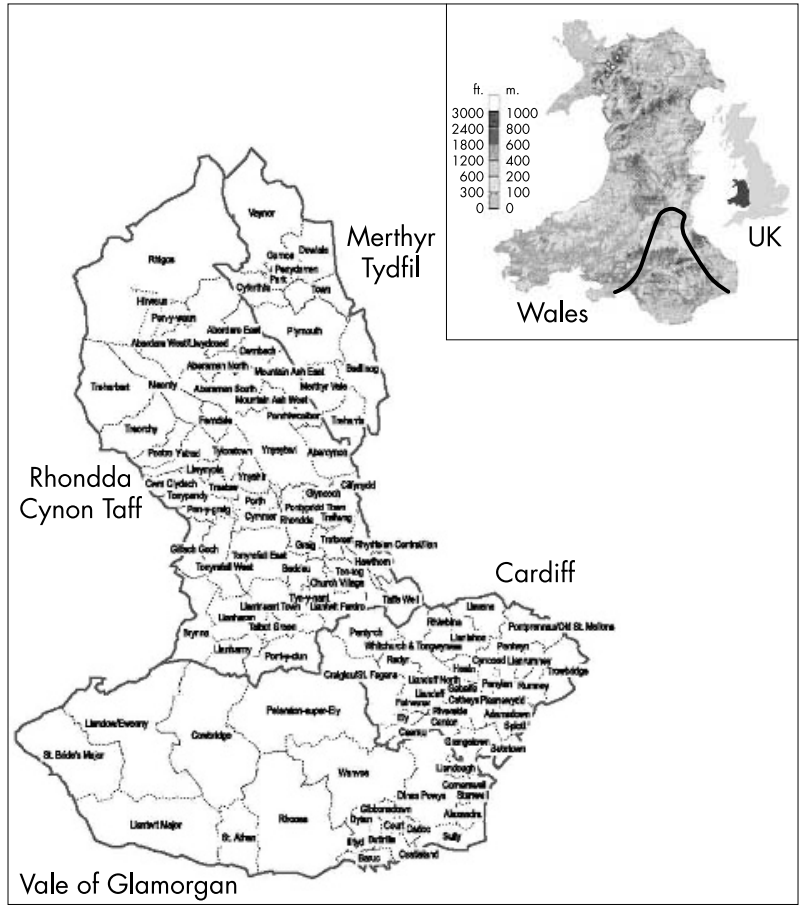

Figure 1 Map of the catchment area of the Bro Taf Health Authority (BTHA) for this population based study with an estimated population of $742400\left(1999^{32}\right.$ ) located in south east Wales, United Kingdom (area demarcated in the inset UK/Wales map).

classed as familial or sporadic LOCA on the basis of available information on the presence or absence of similar disorder or with confirmed pathological genotypes, or history of unexplained gait disturbance in first and second degree relatives. Sporadic cases included those with insufficient family history (lack of family contacts, adoption and early parental deaths) and those with a negative family history.

The exclusion criteria were (i) presence of known acquired ataxia, (ii) ataxic syndrome associated with prominent autonomic dysfunctions and/or atypical parkinsonism suggestive of MSA, (iii) disorders in which ataxia was a minor feature, and (iv) disease duration of $<1$ year. These criteria were set to allow effective comparison with earlier studies adhering to previous definitions for the late onset hereditary ataxias, ${ }^{1}$ MSA, ${ }^{11}$ and the more recent clinico-genetic classification of the ataxias in general. ${ }^{2}$ Patients with LOCA included in the prevalent register and contacted for further assessment included those with previously characterised inherited ataxias and those classified as having ILOCA. For the latter, specific details of history and previous investigations were sought.

\section{Ascertainment of cases, data collection, and statistical analysis}

Ethical approval for the study was obtained from the local research ethics committee. Several ascertainment sources were employed and included (i) records from BTHA and health authority general practices, (ii) departmental databases and personal notifications from regional consultant neurologists over the study period in the Department of Neurology in University Hospital of Wales, and (iii) databases at the Institute of Medical Genetics, Cardiff-both being the referral centres for individuals with LOCA within south east Wales which includes BTHA and three additional neighbouring health authorities. Information was retrieved and stored in accordance with the UK Data Protection Act 1998.
A list of patients with provisional diagnosis of LOCA was constructed from all of these sources and matched against a list of patients provided by the BTHA in a provisional register. This was derived from a computerised search of hospital activity analysis of all patients admitted within BTHA with a diagnosis of "ataxia" according to the 9th and 10th editions of the International Classification of Diseases (ICD). Potential prevalent cases were identified and case notes reviewed to establish the basis for the diagnostic assignment. Patients were grouped as being alive, dead, or unclassified (due to insufficient essential clinical information). The integrated patient list was inspected for duplicate entries, which were then excluded and a preliminary revised register created.

All general practices within the health authority were contacted to verify details of cases where available. The general practitioners (GPs) were also asked to notify any suspected cases within practices and permission was sought to approach patients under their care. GPs who did not reply after three contacts requesting information were considered non-respondents. After obtaining informed consent, patients who agreed to take part in the study underwent more detailed clinical assessment including history (direct and from case notes), clinical examination, and objective measurements of ataxia and activities of daily living (ADL).

A standardised clinical protocol was designed to collect detailed clinical and family history, examination findings, and details of previous investigations. These data were stored in a dedicated database and confidentiality maintained.

Statistical comparisons were made using 95\% confidence intervals (CI) to detect differences in proportions of categorical variables. Calculation of CI using crude rates was based on Poisson's distribution. Pearson's rank correlation coefficient $(r)$ was used to examine correlation between variables.

\section{RESULTS}

A total of 582 cases were identified in the provisional register from multiple sources. Of these, 277 (47.4\%) were from departmental sources, $265(45.8 \%)$ from the computerised search of hospital activity analysis, and 40 (6.8\%) from respondent general practices. A further 106/134 (79.1\%) general practices subsequently responded. After removal of 176 duplicates, a total of 409 cases had been ascertained and included in the preliminary register (fig 2). Of these, 75 $(18.3 \%)$ were dead and $32(7.8 \%)$ were untraceable (mainly due to absence of personal data). Of the remainder, 232/302 (76.9\%) were resident within the BTHA geographic boundary and $70 / 302(23.1 \%)$ were resident outside this boundary on prevalence day and had never resided in the study area. During the period of case ascertainment, we were aware of only one patient who had migrated into the study area. Sixty six $(28.4 \%)$ appeared in more than one source (table 1 ).

The excluded 156/232 (67.2\%) cases comprised 55 (34.5\%) with known acquired ataxia, 2 (1.3\%) with probable or possible MSA, and 99 (63.5\%) who had disorders with ataxia as a minor feature or were cases of non-LOCA $(38 ; 38.8 \%)$ identified with congenital and early onset cerebellar ataxia. These patients were not assessed further. The commonest causes of ataxia $(n=55)$, in order of decreasing frequency were: multiple sclerosis $(19 ; 34.5 \%)$, isolated and familial spastic paraparesis $(13 ; 23.6 \%)$, cerebellar tumours (6; $10.9 \%)$, and alcoholic cerebellar degeneration $(3 ; 5.5 \%)$.

In addition, six incident cases of LOCA were identified within a three year period between 1999 and 2001, providing a mean annual incidence rate of 0.3/100 000 population/year. Of these, one patient had SCA 6 while the remainder were diagnosed as idiopathic. The mean (SD) age of these patients 


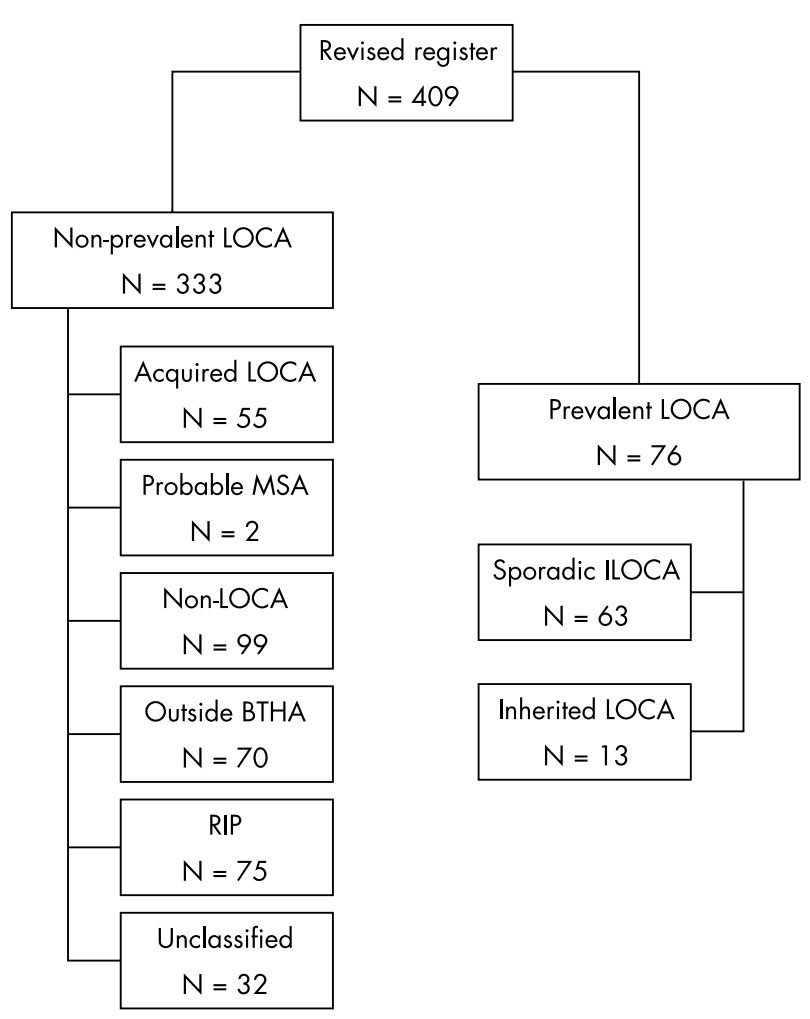

Figure 2 Flow diagram demonstrating the process of recruitment of patients into the prevalent register. RIP, deceased patients.

was $62.3(8.09)$ years (range 51-62) and the sex distribution was equal.

From the initial 409 cases identified in the preliminary register, $333(81.4 \%)$ were excluded (including eight cases who were personally examined). The remaining 76 (18.6\%) were identified as prevalent: 63 with apparently sporadic (ILOCA) and 13 with familial LOCA, in a population of 742 400. Within the familial cases, all probands were unrelated. In seven, no cause has been identified, and six have an established genetic basis: SCA $6(n=2)$; Friedrich's ataxia (FRDA) $(\mathrm{n}=2)$; and dominant episodic ataxias $(n=2)$. Fifty seven $(75 \%)$ prevalent patients were personally examined (54 with ILOCA and three with genetic basis) while 19 cases ( 16 with ILOCA and three with genetic basis) were included in the prevalence estimates on the basis of review of records alone. This information provides crude prevalence rates of 10.2 per 100000 (95\% CI 7.9 to 12.5 ) for amalgamated sporadic and familial LOCA. Prevalence of sporadic, idiopathic LOCA and familial LOCA are shown in table 2 .

Overall, the mean (SD) age of prevalent ILOCA $(n=63)$ patients was 61.79 ( 13.22 ) years (range 35 to 88 ). Forty two patients $(66.7 \%)$ were men. The mean age at onset of symptoms was 53.8 (14.1) years (range 19 to 78 ) with mean disease duration of $8.7(6.3)$ years (range 1 to 31 ). The most frequent presenting symptom was gait disturbance, reported by $49 / 54(90.7 \%)$ patients with ILOCA who were assessed. Of these, 9/49 (18.4\%) also complained of an accompanying symptom of dizziness at some stage after the onset of gait disturbance. The rest of the patients presented with initial symptoms associated with upper limb action tremor or tremulousness and one patient experienced persistent leg cramp prior to onset of gait difficulties. The majority (58; $92 \%)$ of patients with ILOCA declared no relevant family history and no parental consanguinity, and were classed as
Table 1 Comparison of cases ascertained from the various sources*

\begin{tabular}{lcc}
\hline Source & $\begin{array}{c}\text { Preliminary } \\
\text { register }(\%)\end{array}$ & Prevalent register (\%)† \\
\hline Single source & & \\
$\quad$ Neurology databases & $43(18.5)$ & $35(46.1)$ \\
Genetic databases & $9(3.9)$ & $8(10.5)$ \\
General practices & $13(5.6)$ & $5(6.6)$ \\
BTHA & $101(43.5)$ & $18(23.7)$ \\
Multi-source & $66(28.4)$ & $10(13.1)$ \\
Total & 232 & 76 \\
\hline
\end{tabular}

*Majority of prevalent cases were identified from departmental databases (neurology; genetics).

†Amalgamation of sporadic and familial cases of late onset cerebellar ataxia.

BTHA, Bro Taf Health Authority.

sporadic. Of these, five ( $8 \%$ ) cases were considered sporadic because complete pedigree histories were unattainable but no relevant family history was declared. Seven patients reported a family history of late onset progressive ataxia, with undefined genetic basis. In five, the family history was suggestive of autosomal dominant inheritance and two had an apparently recessive pattern.

Two clinical groups were identified within the group of patients with ILOCA: those with a relatively pure cerebellar syndrome $(18 / 54 ; 33.3 \%)$ and those with additional extracerebellar neurological features $(36 / 54 ; 66.7 \%)$. The variable rate of progression, as illustrated by the mean disease duration, shows a significant inverse correlation with the degree of disability $(r=-0.538 ; \mathrm{p}<0.01)$ (fig 3$)$. Based on Barthel's ADL index, 4/54 (7.4\%) patients were fully independent (ADL score 20), 42/54 (77.8\%) had a mild disability (ADL score 15-19), 6/54 (11.1\%) had a moderate disability (ADL score 10-14), and 2/54 (3.7\%) had a severe disability (ADL score 5-9).

\section{DISCUSSION}

In this study, which is the first of its kind in the UK, we describe the clinical phenotype of LOCA in a defined geographical area and discuss the limitations of performing such a study. We have been able to provide prevalence estimates for familial and sporadic LOCA as well as insights into the degree of impairment amongst affected individuals.

Although we believe that the incidence and prevalence data presented here are representative of the region, these figures may be underestimates for a number of reasons. First, complete ascertainment of prevalent patients may not have been achieved since only $79 \%$ of general practices participated, although the yield of prevalent cases from this source was low $(5 / 76 ; 6.6 \%)$. However, we attempted to optimise ascertainment by extending recruitment beyond departmental specialist databases with a community based approach to identify those cases managed solely in a primary care setting, lost to follow up, or misdiagnosed as well as cases seen by other hospital specialists. Such an approach was successful in a community and regional ascertainment in a regional UK study on the prevalence of progressive supranuclear palsy ${ }^{33}$ and is comparable with that of a population adjusted clinical epidemiology (PACE) approach. ${ }^{34}$ However, the efficiency by which individual sources ascertained patients was not directly evaluated by capture-recapture studies.

In addition, the evaluation of the year of diagnosis for prevalent patients revealed clustering within the decade preceding prevalence day (1990s: $n=51 / 76,67 \%$ ) with respect to other decades (1960s: $\mathrm{n}=3 / 76,4 \%$; 1970s: $\mathrm{n}=$ 7/76, 9\%; 1980s: $\mathrm{n}=15 / 76,20 \%)$. This may reflect poor ascertainment of patients diagnosed $\geqslant 10$ years ago. However, 
Table 2 Prevalence of sporadic, idiopathic LOCA (ILOCA) cases in the whole BTHA population (crude and sex specific rates) and prevalence of familial LOCA cases with and without defined genetic loci

\begin{tabular}{|c|c|c|}
\hline Population (BTHA) & Prevalent cases & Estimated prevalence \\
\hline \multicolumn{3}{|l|}{ Sporadic, idiopathic LOCA (ILOCA) cases } \\
\hline Whole $(n=742400)$ & 63 & $\begin{array}{l}\text { Crude rate } 8.4 \text { per } 100000 \\
(95 \% \mathrm{Cl} 7.2 \text { to } 11.6)\end{array}$ \\
\hline Males (all ages) $(n=366800)$ & 42 & $\begin{array}{l}\text { Sex specific rate } 12.5 \text { per } \\
100000(95 \% \mathrm{Cl} 8.9 \text { to } 16.2)\end{array}$ \\
\hline Females (all ages) $(n=375600)$ & 21 & $\begin{array}{l}\text { Sex specific rate } 6.4 \text { per } 100000 \\
(95 \% \mathrm{Cl} 3.8 \text { to } 9.0)\end{array}$ \\
\hline \multicolumn{3}{|l|}{ Inherited LOCA cases } \\
\hline Whole $(n=742400)$ & 13 & $\begin{array}{l}\text { Crude rate } 1.8 \text { per } 100000 \\
(95 \% \mathrm{Cl} 0.8 \text { to } 2.7)\end{array}$ \\
\hline Defined genetic cause $(n=742400)$ & 6 & $\begin{array}{l}\text { Crude rate } 0.81 \text { per } 100000 \\
(95 \% \mathrm{Cl} 0.1 \text { to } 1.5)\end{array}$ \\
\hline Undefined genetic cause $(n=742400)$ & 7 & $\begin{array}{l}\text { Crude rate } 0.9 \text { per } 100000 \\
(95 \% \mathrm{Cl} 0.2 \text { to } 1.6)\end{array}$ \\
\hline
\end{tabular}

accurate mortality data are not currently available for this group of patients and authoritative view from previous authors have estimated the disease duration to be between 10 to 20 years. $^{35}$ If this is confirmed, it may well be compatible with the temporal pattern of the year of diagnosis observed in our patients. Also, although the study was undertaken in a relatively stable population, it is possible that migration of affected patients to the study area may have occurred within the ascertainment period which may have led to an underestimation of the prevalence. This may be particularly relevant in this group of patients for whom treatment is limited and who may not have sought further care following inward migration. However, in the 70 nonprevalent patients identified with LOCA residing in regions neighbouring the study area, there was only one case of inward migration during the period of case ascertainment.

Furthermore, estimates of prevalence can be made in epidemiological studies such as this by multiplying annual disease incidence by two times mean disease duration. Application of this simple formula to our data produces a figure of 5.2 per 100000 (95\% CI 0.7 to 9.7) with wide confidence intervals as a result of the small sample of incident cases for the idiopathic, sporadic LOCA (ILOCA). Our observed figure of 8.4 per 100000 lies within these confidence intervals. The small number of patients with familial basis does not allow us to produce meaningful estimate of prevalence with this method.

Classification of cases as idiopathic could also be a potential confounding factor and might have influenced the diagnostic accuracy in both familial and sporadic cases. The

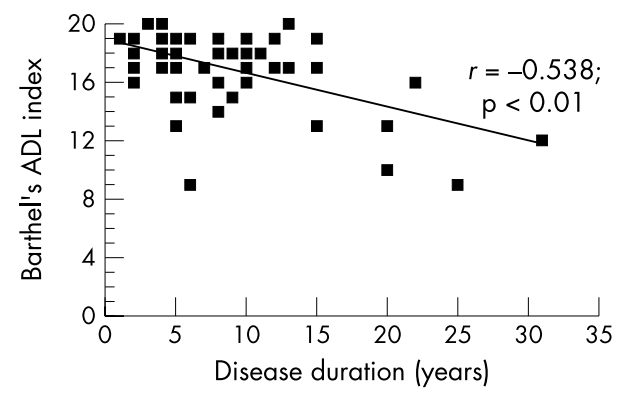

Figure 3 Scatter plot of patients with idiopathic late onset cerebellar ataxia who were assessed showing a significant inverse correlation between the degree of disability (based on Barthel's Index) and the disease duration. ADL, activities of daily living. few incomplete pedigree data obtained from the proband may also have underestimated the prevalence of familial cases. In addition, investigation strategies may vary from one physician to another, as well as over time, even within the same specialist centre. We also recognise that personal review of $25 \%(n=19 / 76)$ of cases in the prevalent register was not possible. However, in each of these cases, clinical assessment had been performed by a neurologist and recorded in the department of neurology notes and clinical data were obtained from this source. Clinical and epidemiological characteristics of those patients personally reviewed and those for whom clinical data were obtained from clinical records were similar.

We set the inclusion and exclusion criteria for prevalent LOCA in the study taking into account both pre and postmolecular LOCA classifications. ${ }^{1211}$ The definition of LOCA in our study allowed us to make comparisons with previously reported prevalence rates of hereditary ataxias. ${ }^{22-27}$ These LOCA-adjusted prevalence rates are shown in table 3. As some of these studies included patients with hereditary spastic paraplegia as well as early onset cerebellar ataxias (including FRDA), these earlier reported figures are likely to be inflated and/or may explain the wide variations observed in previous figures.

The mean age at onset of symptoms of the patients with ILOCA in this study was 53.8 (14.1) years (range 19 to 78), comparable with those reported in other non-geographically defined studies of ILOCA (ranging from 50 to 58.9)..$^{\text {7-9 }}$ Similarly, an unexplained excess of male cases $(2.1: 1)$ has previously been recognised..$^{7}$ We considered that classification based on specific constellation of clinical features was unlikely to be contributory, ${ }^{1}$ so in this series of patients, we delineated in general terms, two groups of ILOCA patients: one group with a relatively pure cerebellar syndrome and the other with extracerebellar features. Other previously reported series had also described the presence of extracerebellar features in over half of cases. ${ }^{78}$ However, it is important to note that longitudinal data may subsequently lead to a change in diagnostic classification for a proportion of patients particularly with respect to MSA.

We identified one case of probable MSA on prevalence day. Subsequent to this, one patient who was previously labelled as sporadic ILOCA also developed features to suggest an alternative diagnosis of MSA. Parkinsonism has been reported to develop in most patients (54-100\%) with MSA while "pure" cerebellar presentation is uncommon (0$16 \%),{ }^{36}$ although this clinical pattern seems to be reversed 
Table 3 Comparison of contemporary prevalence data of inherited ataxias from several countries for the subgroup of LOCA with genetic basis*

\begin{tabular}{lcc}
\hline Reference (country) & $\begin{array}{l}\text { Prevalence rate* } \\
\text { (per } 100 \text { 000) }\end{array}$ & $\begin{array}{l}\text { Predicted prevalence } \\
\text { rate (per 100 000) }\end{array}$ \\
\hline Koeppen et al $1977^{22}$ (UK) & 0.31 & $0.15(95 \% \mathrm{Cl} 0.03$ to 0.27$)$ \\
Sridharan ef al $1985^{27}$ (Libya) & 4.8 & $2.1(95 \% \mathrm{Cl} 0.8$ to 3.42$)$ \\
Orozco et al $1989^{25}$ (Cuba) & 41 & $22.3(95 \% \mathrm{Cl} 15.6$ to 29$)$ \\
Polo et al $1991^{26}$ (Spain) & 20.2 & $3.3(95 \% \mathrm{Cl} 1.7$ to 4.9$)$ \\
Leone et al $1995^{23}$ (ltaly) & 14.8 & $8.7(95 \% \mathrm{Cl} 3$ to 14.4$)$ \\
Current study & 1.8 & $1.8(95 \% \mathrm{Cl} 0.8$ to 2.7$)$ \\
\hline
\end{tabular}

in Japanese cases. ${ }^{37}$ This is supported by the fact that contemporary epidemiological studies of MSA employ search criteria for MSA using terms associated with "parkinsonism" rather than with cerebellar ataxia. ${ }^{38}$ However, some MSA patients may present solely with features of cerebellar ataxia leading to an initial diagnosis of ILOCA or olivopontocerebellar atrophy (OPCA) prior to the onset of defining features of MSA. In contrast to OPCA, the term ILOCA makes no presumptions about the status of extracerebellar pathology. Recent longitudinal data have suggested that up to one-third of cases with ILOCA may develop into MSA. ${ }^{5}{ }^{10}$ Therefore, in our study, there is a possibility that among this series of patients, a proportion might evolve into MSA and contaminate the accuracy of our reported prevalence rate. Longitudinal population based data are required to evaluate this further.

Our study also highlights the generally progressive nature of this disorder, although a variable degree of disability and rate of disease progression was noted among the cases. The majority of patients $(\mathrm{n}=42 / 54 ; 77.8 \%)$ had an ADL score of 15-19 suggesting a mild degree of disability, irrespective of variability in the disease duration. The majority remained ambulant (92\%) but often employed a walking aid. Two patients with an ADL score of 9 or less (severe disability) and disease duration longer than 20 years had lost the autonomous ability to walk. Among this series of patients, 49/54 $(90.7 \%)$ admitted to dependency on regular daily assistance from a carer (such as close relatives or employee of social services) with the rest being independent.

In conclusion, we estimated the prevalence of idiopathic and familial LOCA using a population based approach, and have given further information on the clinical phenotype. These data provide further guidance to clinicians on aspects of counselling and prognosis for patients with ILOCA. This may also inform the appropriate application of genetic and immunological investigations in cases previously considered to be idiopathic. In addition, we have also created opportunities for longitudinal assessment of clinical features, impairment, impact on quality of life as well as the framework to explore the poorly understood relationship between MSA and ILOCA.

\section{ACKNOWLEDGEMENTS}

We wish to extend our gratitude to the staff and consultant colleagues at the Section of Neurology, University Hospital of Wales (G Llewelyn, INF McQueen, TAT Hughes, PEM Smith, FJ Thomas, L Johns, and L Coates), the staff at the Institute of Medical Genetics (J Sampson, L Lazarou, R Butler, J Myring, L Meredith, Q Roberts, M McDonald, and R Brito), the staff at the medical records library at University Hospital of Wales, and the participating general practitioners for their support.

\section{Authors' affiliations}

M B Muzaimi, J Thomas, C M Wiles, N P Robertson, Section of Neurology, Department of Medicine, University Hospital of Wales, Cardiff, UK
S Palmer-Smith, L Rosser, P S Harper, D Ravine, Institute of Medical Genetics, University Hospital of Wales, Cardiff, UK

JT was funded by the Welsh Office Research and Development (WORD) and MBM is supported by School of Medical Sciences, University Science Malaysia (USM).

Competing interests: none declared

\section{REFERENCES}

1 Harding AE. The hereditary ataxias and related disorders. New York: Churchill Livingstone, 1984.

2 Klockgether T. Handbook of Ataxia Disorders. New York: Mercel-Dekker, 2000.

3 Gwinn-Hardy KA, Evidente VG, Caviness JN, et al. Hereditary ataxias. Mayo Clin Proc 2000;75:475-90.

4 Moseley ML, Benzow KA, Schut $\sqcup$, et al. Incidence of dominant spinocerebellar and Friedreich triplet repeats among 361 ataxia families. Neurology 1998;51:1666-71.

5 Schols L, Szymanski S, Peters S, et al. Genetic background of apparently idiopathic sporadic cerebellar ataxia. Hum Genet 2000;107:132-7.

6 Schapira AH. Mitochondrial disorders. Curr Opin Neurol 2000;13:527-32.

7 Abele M, Burk K, Schols L, et al. The aetiology of sporadic adult-onset ataxia. Brain 2002; 125(Pt 5):961-8.

8 Harding AE. "Idiopathic" late onset cerebellar ataxia: A clinical and genetic study of 36 cases. J Neurol Sci 1981;51:259-71.

9 Klockgether T, Burk K, Wullner U, et al. Risk factors for idiopathic cerebellar ataxia of late onset. J Neurol Sci 1998;160:171-4.

10 Gilman S, Little R, Johanns J, et al. Evolution of sporadic olivopontocerebellar atrophy into multiple system atrophy. Neurology 2000;55:527-32.

11 Gilman S, Low PA, Quinn N, et al. Consensus statement on the diagnosis of multiple system atrophy. J Neurol Sci 1999;163:94-8.

12 Basu P, Chattopadhyay B, Gangopadhaya PK, et al. Analysis of CAG repeats in SCA1, SCA2, SCA3, SCA6, SCA7 and DRPLA loci in spinocerebellar ataxia patients and distribution of CAG repeats at the SCA1, SCA2 and SCA6 loci in nine ethnic populations of eastern India. Hum Genet 2000; 106:597-604.

13 Filla A, De Michele G, Santoro L, et al. Spinocerebellar ataxia type 2 in southern Italy: a clinical and molecular study of 30 families. J Neurol 1999;246:467-71.

14 Leggo J, Dalton A, Morrison PJ, et al. Analysis of spinocerebellar ataxia types $1,2,3$, and 6, dentatorubral-pallidoluysian atrophy, and Friedreich's ataxia genes in spinocerebellar ataxia patients in the UK. J Med Genet 1997;34:982-5.

15 Maruyama H, Izumi $Y$, Morino $H$, et al. Difference in disease-free survival curve and regional distribution according to subtype of spinocerebellar ataxia: a study of 1286 Japanese patients. Am J Med Genet 2002; 114:578-83

16 Nagaoka U, Suzuki Y, Kawanami T, et al. Regional differences in genetic subgroup frequency in hereditary cerebellar ataxia, and a morphometrical study of brain MR images in SCA1, MJD and SCA6. J Neurol Sci 1999; 164:187-94

17 Pujana MA, Corral J, Gratacos M, et al. Spinocerebellar ataxias in Spanish patients: genetic analysis of familial and sporadic cases. The Ataxia Study Group. Human Genetics 1999;104:516-22.

18 Ranum LP, Lundgren JK, Schut $\amalg$, et al. Spinocerebellar ataxia type 1 and Machado-Joseph disease: incidence of CAG expansions among adult-onset ataxia patients from 311 families with dominant, recessive, or sporadic ataxia. Am J Hum Genet 1995:57:603-8.

19 Schols L, Amoiridis G, Buttner T, et al. Autosomal dominant cerebellar ataxia: Phenotypic differences in genetically defined subtypes? Ann Neurol 1997;42:924-932.

20 Storey $E$, du Sart D, Shaw JH, et al. Frequency of spinocerebellar ataxia types $1,2,3,6$, and 7 in Australian patients with spinocerebellar ataxia. Am J Med Genet 2000;95:351-7.

21 Silveira I, Lopes-Cendes I, Kish S, et al. Frequency of spinocerebellar ataxia type 1, dentatorubropallidoluysian atrophy, and Machado-Joseph disease mutations in a large group of spinocerebellar ataxia patients. Neurology 1996;46:214-18

22 Koeppen AH, Hans MB, Shepherd DI, et al. Adult-onset hereditary ataxia in Scotland. Arch Neurol 1977;34:611-18. 
23 Leone M, Bottacchi E, Dalessandro G, et al. Hereditary ataxias and paraplegias in Valle d'Aosten, Italy: a study of prevalence and disability. Acta Neurol Scand 1995;91:183-7.

24 Mori M, Adachi Y, Kusumi M, et al. A genetic epidemiological study of spinocerebellar ataxias in Tottori prefecture, Japan. Neuroepidemiology 2001;20:144-9.

25 Orozco G, Estrada R, Perry TL, et al. Dominantly inherited olivopontocerebellar atrophy from eastern Cuba. Clinical, neuropathological, and biochemical findings. J Neurol Sci 1989;93:37-50

26 Polo JM, Calleja J, Combarros O, et al. Hereditary ataxias and paraplegias in Cantabria, Spain. An epidemiological and clinical study. Brain $1991 ; 114(\mathrm{Pt} 2): 855-66$.

27 Sridharan R, Radhakrishnan K, Ashok PP, et al. Prevalence and pattern of spinocerebellar degenerations in northeastern Libya. Brain 1985; 108(Pt 4):831-43.

28 Harper PS, Tyler A, Smith S, et al. A genetic register for Huntington's chorea in South Wales. J Med Genet 1982;19:241-5.

29 Swingler RJ, Compston DA. The prevalence of multiple sclerosis in south east Wales. J Neurol Neurosurg Psychiatry 1988:51:1520-4.

30 MacMillan JC, Harper PS. Single-gene neurological disorders in South Wales: an epidemiological study. Ann Neurol 1991;30:411-14.
31 James CM, Harper PS, Wiles CM. Motor neurone disease: a study of prevalence and disability. QJM 1994;87:693-9.

32 Office of National Statistics. A Statistical Focus on Wales. London: ONC 1999:1-4.

33 Nath U, Ben-Shlomo Y, Thomson RG, et al. The prevalence of progressive supranuclear palsy (Steele-Richardson-Olszewski syndrome) in the UK. Brain $2001 ; 124(\mathrm{Pt} 7): 1438-49$.

34 Proctor SJ, Taylor PR. A practical guide to continuous population-based data collection (PACE): a process facilitating uniformity of care and research into practice. QJM 2000;93:67-73.

35 Klockgether T, Schroth G, Diener HC, Dichgans J. Idiopathic cerebellar ataxia of late onset: natural history and MRI morphology. I Neurol Neurosurg Psychiatry 1990;53:297-305.

36 Burn DJ, Jaros E. Multiple system atrophy: cellular and molecular pathology. Mol Pathol 2001:54:419-26.

37 Watanabe $\mathbf{H}$, Saito $\mathrm{Y}$, Terao $\mathrm{S}$, et al. Progression and prognosis in multiple system atrophy: an analysis of 230 Japanese patients. Brain 2002; 125:1070-83.

38 Schrag A, Ben-Shlomo Y, Quinn NP. Prevalence of progressive supranuclear palsy and multiple system atrophy: a cross-sectional study. Lancet 1999;354:1771-5.

\section{Making Health Care Safer 2004}

21-22 October 2004

Royal College of Physicians, London

A two day conference for all professionals dedicated to providing safer health care for all.

Register now! Early booking discount available.

See website for details: www.quality.bmipg.com 